\title{
Acknowledgement to Reviewers of Catalysts in 2016
}

\author{
Catalysts Editorial Office \\ Published: 11 January 2017 \\ MDPI AG, St. Alban-Anlage 66, 4052 Basel, Switzerland; catalysts@mdpi.com
}

The editors of Catalysts would like to express their sincere gratitude to the following reviewers for assessing manuscripts in 2016.

We greatly appreciate the contribution of expert reviewers, which is crucial to the journal's editorial process. We aim to recognize reviewer contributions through several mechanisms, of which the annual publication of reviewer names is one. Reviewers receive a voucher entitling them to a discount on their next MDPI publication and can download a certificate of recognition directly from our submission system. Additionally, reviewers can sign up to the service Publons (https://publons.com) to receive recognition. Of course, in these initiatives we are careful not to compromise reviewer confidentiality. Many reviewers see their work as a voluntary and often unseen part of their role as researchers. We are grateful to the time reviewers donate to our journals and the contribution they make.

If you are interested in becoming a reviewer for Catalysts, see the link at the bottom of the webpage http://www.mdpi.com/reviewers.

The following reviewed for Catalysts in 2016:

Abad, Enrique

Abdala, Paula Macarena

Abitbol, Tiffany

Abu-Reziq, Raed

Agustín, Bueno-López

Alayoglu, Selim

Albini, Angelo

Alegre, Cinthia

Aléman, José

Alemany Arrebola, Luís José

Alonso, Diego A.

Alt, Helmut G.

Álvarez Moreno, Andrea

Amadelli, Rossano

Amarasekara, Ananda S.

Ampelli, Claudio

Andersen, Shuang Ma

Ansorge-Schumacher, Marion

Antolini, Ermete

Arcoya Martín, Adolfo

Arena, Francesco

Arends, Isabel W. C. E.

Arias, Pedro L.

Ariga, Katsuhiko

Arnold, Ulrich

Aubé, Jeffrey
Ho, Wing-Kei

Hohn, Keith

Höhne, Matthias

Horiuchi, $\mathrm{Yu}$

$\mathrm{Hu}$, Anming

Huang, Wenyu

Hunger, Michael

Husson, Jérôme

Ibrahim, Hussameldin

Ichihashi, Yuichi

Iglesias, Marta

Iizuka, Yasuo

Ikenaga, Na-oki

Imai, Hiroyuki

Imanishi, Akihito

Inoue, Hiroshi

ISHIDA, Tamao

Ishiguro, Hitoshi

Ivanov, Ivan

Jacobsen, Elisabeth Egholm

Jaffe, Eileen

Jang, Ho Won

Jang, Hye-Young

Jee, Youngseok

Jensen, Anker Degn

Jeon, Seokwoo
Palomo, Claudio

Palzer, Stefan

Pansare, Sunil V.

Pant, Deepak

Papadopoulou, Christina

Park, Soo-Jin

Park, Tae Joo

Park, Young-kwon

Parlett, Christopher

Pasini, Dario

Passaglia, Elisa

Patterson, Howard H.

Pawelec, Barbara

Pedro, José R.

Pedro, Maireles-Torres

Pedrosa, Rafael

Pellegrino, Francesco

Peral, José

Pereira, Luciana

Perez-Jimenez, Raul

Periana, Roy A.

Petit, Christophe

Petrou, Panagiota S.

Pettersson, Lars J.

Pigge, F. Christopher

Pillai, Suresh 
Augustine, Robert

Auroux, Aline

Ayastuy Arizti, José Luis

Baba, Toshihide

Bakker, Martin G.

Bálint, Erika

Ball, Zachary

Bandaru, Prabhakar R.

Banham, Dustin

Bartholomew, Calvin

Bavaro, Teodora

Beato, Pablo

Bechelany, Mikhael

Bellardita, Marianna

Benco, Lubomir

Bensaid, Samir

Bentrup, Ursula

Bhagavathy, Ganga V.

Bhargava, Suresh

Biasi, Pierdomenico

Bignozzi, Carlo Alberto

Blanco, Rosa M.

Bolivar, Juan

Bond, Jesse Q.

Bontemps, Sebastien

Borole, Abhijeet P.

Brenna, Elisabetta

Bui, Hao Van

Cabot, Pere-Lluís

Cacciato, Giuseppe

Cadierno, Victorio

Camblor, Miguel A.

Campisi, Sebastiano

Candeias, Nuno R.

Carabineiro, Sónia A. C.

Carboni, Michael

Carmona, Daniel

Carreon, Moises

Cassani, Maria Cristina

Castanheiro, José

Castaño, Pedro

Castoldi, Lidia

Cecilia, Juan Antonio

Centeno, Miguel Angel

Cesano, Federico

Chan, Kin Shing

Chang, Chang-Tang

Chang, Chia-En A.

Chang, Chi-jung

Charnay, Clarence

Chauvin, Jérôme

Chavez, Ferman
Jiang, Yi

Jo, Young Min

Jones, David

Jones, Matthew D.

Jradi, Khalil

Jung, Jongwan

Jung, Kwang-deog

Kadokawa, Jun-ichi

Kalantar Zadeh, Kourosh

Kamata, Hiroyuki

Kamegawa, Takashi

Kameyama, Hideo

Kamiya, Kazuhide

Kanaev, Andrei

Kaneda, Kiyotomi

Kaneko, Hiromasa

Kapteijn, Freek

Katapodis, Petros

Kato, Keisuke

Katryniok, Benjamin

Katsaounis, Alexandros

Katsumata, Hideyuki

Kawanami, Hajime

Kayaki, Yoshihito

Kee, Robert J.

Keum, Young Soo

Kim, Hoon

Kim, Hyung Kwoun

Kim, Hyunwoo

Kim, Jae Chang

Kim, Jinho

Kim, Junbom

Kim, Sang Hoon

Kim, Sooyoung

Kim, T. Doohun

Kim, Younghun

Kim, Yu Kwon

Kirillov, Evgueni

Kiss, János

Kitamura, Tsugio

Kiwi-Minsker, Lioubov

Klages, Claus-Peter

Kleij, Arjan W.

Koenig, Daniel H.

Kohl, Paul A.

Köhler, Klaus

Kondrat, Simon

Konkolewicz, Dominik

Konsolakis, Michalis

Kopylovich, Maximilian

Koschorreck, Katja

Kourist, Robert
Pinilla Ibarz, José Luis

Pino, Lidia

Pitsikalis, Marinos

Plakas, Konstantinos

Plasseraud, Laurent

Poater, Albert

Polfus, Jonathan M.

Politano, Antonio

Pollet, Eric

Poulos, Thomas L.

Prandi, Cristina

Prata, José V.

$\mathrm{Pu}$, Ying-Chih

Qiu, Kaipei

Rackemann, Darryn

Rafael Ruiz, Jose'

Ram, Manoj K.

Ramanathan, Anand

Ramis, Gianguido

Ramón, Diego

Ranjan, Chinmoy

Raspoli, Anna

Rataboul, Franck

Ravelli, Davide

Razavi, Pedram

Reddy, Amaranatha

Redshaw, Carl

Reetz, Manfred T.

Reiser, Oliver

Renaud, Jean-Luc

Reshetenko, Tatyana V.

Richards, Nigel

Rimoldi, Luca

Rizzi, Gian Andrea

Roberto, Dominique

Rodriguez, Jose

Rodríguez-Castellón, Enríque

Rodríguez-Ramos, Inmaculada

Roduner, Emil

Roglans, Anna

Rojas, Sergio

Romanias, Manolis

Roper, Donald Keith

Rousset, Jean-Luc

Rtimi, Sami

Rubiolo, Juan

Rupprechter, Günther

Saidi, Wissam A.

Saito, Nagahiro

Sanchez, David V. P.

Sanjust, Enrico

Sannino, Diana 
Chen, Bing-Hung

Chen, Chia-Yun

Chen, Chiing-Chang

Chen, Feng You

Chen, Hua-Wei

Chen, Hui-Lung

Chen, Jinwen

Cheng, Kong-Wei

Chiang, Yin-Ru

Chinchilla, Rafael

Chmielarz, Lucjan

Cho, Moo H.

Choi, Sung-Min

Christoforidis, Konstantinos C.

Christos, Malliakas

Chrysina, Evangelia D.

Chul Wee, Lee

Chundawat, Shishir P. S.

Cirillo, Giuseppe

Claver, Carmen

Cole, Matt

Concepción, Patricia

Coto, Baudilio

Cozzi, Piergiorgio

Crabtree, Robert H.

Creager, Stephen E.

Cristiani, Pierangela

Croatt, Mitchell P.

Crocker, Mark

Dal Santo, Vladimiro

Davies, Philip

Daza, Yolanda

Deagostino, Annamaria

Del Amo, Vicente

Del Hierro, Isabel

Delahay, Gerard

Delaude, Lionel

Delferro, Massimiliano

Delidovich, Irina

Desmet, Tom

Dhakshinamoorthy, Amarajothi

Dhudshia, Bhartesh

Di Bartolomeo, Antonio

Di Noto, Vito

Díez, David

Dijkhuizen, Lubbert

Dimitratos, Nikolaos

Dolzhenko, Anton V.

Domine, Marcelo Eduardo

Donat, Felix

Dong, Mingdong

Duarte, Fernanda G.
Kozhevnikov, Ivan V.

Krempner, Clemens

Kubota, Yoshihiro

Kusada, Kohei

Laali, Kenneth

Ladero, Miguel

Ladero Galán, Miguel

Laguna, Oscar Hernando

Lai, Yi Sheng

Lamonier, Jean-François

Lancaster, Simon J.

Landi, Gianluca

Larabi, Cherif

Lavacchi, Alessandro

Lavandera, Ivan

Leclerc, Corey

Lecomte, Philippe

Lee, Chien-Liang

Lee, Hyuck Mo

Lee, Hyunjoo

Lee, Ilkeun

Lee, Yong Rok

Lefebvre, Frederic

Lefebvre, Olivier

Lefferts, Leon

Léger, Bastien

Lei, Yong

Lemonidou, Angeliki A.

Lenarda, Maurizio

Leonard, Brian

Leyva-Pérez, Antonio

Lianos, Panagiotis

Likodimos, Vlassios

LIM, Yee Fun

Lin, Long-Liu

Ling, Yong-Chien

Lisi, Luciana

Litvić, Mladen

Liu, Dongxia

Liu, Jun

Liu, Shiuh-Tzung

Liu, Yanbiao

Llorca, Jordi

Lobato, Justo

Lofrano, Giusy

Long, Nguyen

Longhi, Mariangela

Lopez, Nuria

Lopez Nieto, José Manuel

Lopez-Serrano, Joaquin

Luber, Sandra

Lugemwa, Fulgentius Nelson
Sanz Díez, Roberto

Sapi, Janos

Sasaki, Takehiko

Sato, Kazuhiko

Sato, Takafumi

Saviano, Michele

Sawabe, Kyoichi

Scirè, Salvatore

Seayad, Jayasree

Sebastián, David

Secundo, Francesco

Selli, Elena

Sémeril, David

Seo, Hyo Jin

Sergeev, Alexey

Seshan, Kulathuiyer

Seto, Shigeki

Shalom, Menny

Shanmugam, Sangaraju

Shibasaki, Masakatsu

Shih, Shao-Ju

Shinada, Tetsuro

Shiraishi, Yasuhiro

Shokouhimehr, Mohammadreza

Shon, Young-Seok

Shul, Yong-gun

Sicilia, Emila

Siddiki, S. M. A. Hakim

Silvério, Sara C.

Simón, Luis

Simons, Thomas

Skretas, Georgios

Smith, Michael A.

Snapper, Marc

Sohn, Youngku

Solans-Monfort, Xavier

Solsona, Benjamín

Son, Seung Uk

Song, Young Chae

Sotiropoulos, Sotirios

Specchia, Stefania

Spormann, Alfred M.

Steinfeldt, Norbert

Sterner, Reinhard

Stratakis, Manolis

Strunk, Jennifer

Suárez, Silvia

Sugai, Takeshi

Suganuma, Satoshi

Sugi, Yoshihiro

Sugiyama, Shigeru

Suib, Steven L. 
Dujardin, Christophe

Duprez, Daniel

Easton, Brad

Eigenberger, Gerhart

Elkasabi, Yaseen

Engels, Bernd

Engle, Keary M.

Ereña, Javier

Estrine, Boris

Faba, Laura

Faccio, Greta

Fajín, José L. C.

Falaras, Polycarpos

Farrauto, Robert J.

Fattuoni, Claudia

Fehrmann, Rasmus

Feliz, Marta

Fernandes, Pedro

Fernández, Francisco J.

Fernandez-Lafuente, Roberto

De Filippis, Paolo

Flores, Juan C.

Flox, Cristina

Fochi, Mariafrancesca

Fodor, Csaba

Fornasiero, Paolo

Forzatti, Pio

Fraile, José M.

Franssen, Maurice C. R.

Frediani, Piero

Fromm, Katharina

Frongia, Angelo

$\mathrm{Fu}$, Yen-pei

Fujihara, Tetsuaki

Fullana, Andres

Furube, Akihiro

Furusawa, Takeshi

Compton, Richard

Gaczynska, Maria

Gaigneaux, Eric

Gakhar, Ruchi

Galán-Mascarós, José Ramón

Galletti, Anna Maria Raspolli

Galvita, Vladimir V.

Ganter, Christian

Gao, Pu-Xian

Gao, Wei

Gao, Zhiqiang

García, Juan

Gardossi, Lucia

Gatti-Lafranconi, Pietro

Gayubo, Ana G.
Lundgren, Edvin

Luscombe, Christine K.

Macario, Anastasia

Macone, Alberto

Maestri, Matteo

Mahrwald, Rainer

Maiyalagan, Thandavaryan

Mäki-Arvela, Päivi

Makio, Haruyuki

Maksimovich, Nelly

Malardier-Jugroot, Cecile

Marchionni, Andrea

Maria Luisa, Contreras

Marinkovic, Nebojsa

Marino, Tiziana

Marnellos, George

Marotta, Raffaele

Marqués-López, Eugenia

Márquez, Francisco

Martin, Jessica

Martinez Arias, Arturo

Martins, Luísa M. D. R. S.

Mase, Nobuyuki

Masterson, Douglas

Matam, Santhosh

Matsui, Daisuke

Matsuoka, Shin-ichi

Mauriello, Francesco

Mayoral, Alvaro

Mazzei, Pierluigi

Medio-Simón, Mercedes

Mei, Bastian

Menendez, Rosa

Merlini, Lucio

Meroni, Daniela

Meunier, Frédéric $C$.

Meyer, Randall J.

Mignani, Adriana

Milanesio, Marco

Millet, Jean-Marc M.

Minakshi, Manickam

Mino, Takashi

Miseki, Yugo

Mishra, Yogendra Kumar

Mitran, Gheorghita

Miura, Tsuyoshi

Miyamoto, Manabu

Mohr, Fabian

Mok, Young Sun

Mokaya, Robert

Moliner, Manuel

Moliner, Vicent
Sun, Hongqi

Sunna, Anwar

Szostak, Michal

Taddei, Maurizio

Takagi, Yasumasa

Takeda, Youhei

Takeuchi, Daisuke

Takuya, Koseki

Tanaka, Shinji

Tartakovsky, Leonid

Teng, Hsisheng

Ter Heijne, Annemiek

Terreni, Marco

Testa, Maria Luisa

Testino, Andrea

Thybaut, Joris W.

Tian, Shiliang

Tomita, Koji

Toops, Tod

Topakas, Evangelos

Totani, Kiichiro

Toulhoat, Herve

Toyao, Takashi

Trapp, Oliver

Trincone, Antonio

Trzeciak, Anna M.

Tsai, Fu-Yu

Tsai, Shau-Wei

Uchida, Hiroyuki

Ueda, Mitsuhiro

Uemiya, Shigeyuki

Ushiyama, Hiroshi

Vaiano, Vincenzo

Valange, Sabine

Valente, Artur

Van Sint Annaland, Martin

Vedrine, Jacques

Velasco-Velez, Juan J.

Venieri, Danae

Venvik, Hilde

Dimitra, Papadaki

Vidal, Hilario

Vilé, Gianvito

Villa, Alberto

Vizcaíno, Arturo J.

Vlachos, Dimitrios

Wachs, Israel

Wang, Qun

Wang, Shaobin

Wark, Michael

Waser, Mario

Watanabe, Masaru 
Ge, Xiaoming

Geletii, Yurii V.

Genty, Eric

Genuino, Homer C.

Gervasini, Antonella

Getsoian, Andrew (Bean)

Ghosh, Anindya

Gil Bravo, Antonio

Gilarranz, Miguel A.

Glaser, Roger

Gomes, José Richard Baptista

Gómez, Montserrat

González-Marcos, María P.

Gotor-Fernández, Vicente

Grisi, Fabia

Grünert, Wolfgang

Grunwald, Ingo

Guerrero, Antonio

Guidotti, Matteo

Guisan, Jose Manuel

Gupta, Sanju

Ha, Donhyung

$\mathrm{Ha}$, Kyoung-Su

Ha, Phil Heon

Haan, John L.

Ham, Hyung Chul

Hamad-Schifferli, Kimberly

Hanaor, Dorian

Haouas, Mohamed

Hara, Kenji

Hara, Michikazu

Hargreaves, Justin

Hartmann, Nils

Harvey, Benjamin G.

Hashimoto, Makoto

Hashmi, A. Stephen K.

Haunschild, Robin

Hayashi, Takashi

Haynes, Anthony

Heiskanen, Juha

Helsch, Gundula

Herrera, Concepcion

Hervés-Beloso, Pablo

Higashi, Masanobu

Hikichi, Shiro

Hisada, Kenji
Monflier, Eric

Morales, Gabriel

Moran, Joseph

Moreno, Jovita

Moreno Tost, Ramón

Morgenstern, Ralf

Mori, Kohsuke

Morita, Nobuyoshi

Mossin, Susanne

Moyano, Albert

Muench, Falk

Mukarakate, Calvin

Munoz, Macarena

Murai, Toshiaki

Murcia-López, Sebastián

Murzin, Dmitry Yu.

Nagaoka, Hiroyuki

Nahm, Kee Suk

Nakagawa, Yoshinao

Nakashima, Naotoshi

Nakata, Norio

Nambo, Masakazu

Nemoto, Koji

Neophytides, Stylianos

Nguyen, Hien

$\mathrm{Ni}$, Meng

Nieken, Ulrich

Nieto, Lucía García

Nikitenko, Sergey I.

Nishikiori, Hiromasa

Nishiwaki, Nagatoshi

Notestein, Justin

Nova, Isabella

Nypelo, Tiina

O'Neill, Ellis

Oakhill, Jonathan S.

Ochiai, Tsuyoshi

Oh, Jae-Min

Oh, Jihun

Ohtani, Bunsho

Ortuno, Manuel A.

Palma, Vincenzo

Palmas, Simonetta

Palmer, David

Palmer, James

Palmieri, Gianna
Weckhuysen, Bert

Weidenkaff, Anke

Whittlesey, Michael

Wilde, Nicole

Wilhelm, Rene

Wiśniewski, Marek

Wohlrab, Sebastian

Wong, Dominic W. S.

Woo, Hee Chul

Wood, Joe

Wu, Ho-Shing

Wu, Kevin

Xafenias, Nikolaos

$\mathrm{Xu}$, Bingjun

Xuan, Jin

Yabushita, Mizuho

Yamamoto, Yoshihiko

Yan, Kai

Yan, Ning

Yang, Dong

Yang, Kap

Yasumori, Atsuo

Yentekakis, Ioannis V.

Yerushalmi, Roie

Yoon, Wang Lai

Yoshida, Ken-ichi

Yoshimoto, Makoto

Younis, Adnan

Yu, Jaecheul

Yung, Matthew M.

Yurchenko, Olena

Zaccheria, Federica

Zandi, Omid

Zangari, Giovanni

Zeczycki, Tonya

Zhang, Haifei

Zhang, Hui

Zhang, Jian

Zhang, Liang

Zhao, Xianhui

Zhou, Pei

Zhu, Chengzhou

Zidki, Tomer

Zong, Yun

Zakin, Jacques L. 